\title{
Recent advances in combined wireless power and data transfer
}

\author{
Henry W Boger* \\ Consultant, Senior Member of the International Society of Automation, Birmingham, Hoover, USA
}

\section{Introduction}

This introduction summarizes the work of various scholars in this important area [1-5].

In general, wireless power transfer from a power source to a receiver is implemented through electromagnetic propagation [6]. Similar to wireless information transmission, wireless power transfer also suffers from propagation loss, including path loss, shadowing and fast fading. Therefore, transfer efficiency is a critical and challenging issue for wireless power transfer. To solve this problem, multi-antenna techniques are introduced into wireless power transfer to improve the transfer efficiency by using energy beamforming. In [7], the design methods of the optimal transmit beam for power transfer were given in MIMO broadcast systems. Furthermore, considering the imperfect channel state information at the power source, a robust energy beamforming strategy was proposed in [8]. However, due to the limited number of antennas at the power source, the energy efficiency based on the traditional multi- antenna systems is difficult to satisfy the practical requirement, especially when the transfer distance is not so short [9]. Recently, large-scale MIMO deploying a huge number of antenna at the transmitter is proposed to enormously improve the transmission performance by exploiting its large array gain.

Intuitively, the ultimate purpose of wireless power transfer is to fulfill the need of the receiver for work. As a simple example, in medical field, the implanted equipment in body is powered through wireless power transfer, and then transmits the medical data to the outside receiver with the harvested energy. However, most of previous analogous works analyze and design the wireless power transfer without taking into consideration the utilization of the harvested energy. In this letter, we consider joint wireless information and power transfer in large-scale MIMO systems employing energy beamforming, where the power receiver transmits the information with the harvested energy, namely wireless powered communication. Since energy harvesting and information transmission are impossibly carried out simultaneously, time slot should be divided into the harvesting and the transmitting components. In order to optimize the performance, it is necessary to determine the optimal time switch point, namely time resource allocation. Moreover, transmit power at the power source, as another important resource, also affects the ultimate performance. Recently, energy-efficient communications, namely green communication, gain more and more prominence due to energy shortage and greenhouse effect [12]. This letter focuses on the maximization of energy efficiency, defined as the delivered information bits per Joule harvested energy, by jointly optimizing transfer duration and transmit power. The contributions of this letter lie in three folds. First, it solves the challenging problem of long-distance wireless information and power transfer. Second, it improves the energy efficiency enormously. Third, it provides high QoS guarantee.

A robust energy beamforming strategy was proposed in [8]. However, due to the limited number of antennas at the power source, the energy efficiency based on the traditional multi- antenna systems is difficult to satisfy the practical requirement, especially when the transfer distance is not so short [9]. Recently, large-scale MIMO deploying a huge number of antenna at the transmitter is proposed to enormously improve the transmission performance by exploiting its large array gain $[10,11]$. For example, in the $60 \mathrm{GHz} \mathrm{WiFi} 802.11 / \mathrm{ac}$, since small size antenna is possible, large-scale antenna beamforming is exactly what is being implemented. Similarly, large-scale MIMO can be used to effectively improve the performance of wireless power transfer.

Intuitively, the ultimate purpose of wireless power transfer is to fulfil the need of the receiver for work. As a simple example, in medical field, the implanted equipment in body is powered through wireless power transfer, and then transmits the medical data to the outside receiver with the harvested energy. However, most of previous analogous works analyse and design the wireless power transfer without taking into con- sideration the utilization of the harvested energy. In this letter, we consider joint wireless information and power transfer in large-scale MIMO systems employing energy beamforming, where the power receiver transmits the information with the harvested energy, namely wireless powered communication. Since energy harvesting and information transmission are impossibly carried out simultaneously, time slot should be divided into the harvesting and the transmitting components. In order to optimize the performance, it is necessary to determine the optimal time switch point, namely time resource allocation. Moreover, transmit power at the power source, as another important resource, also affects the ultimate performance. Recently, energy-efficient communications, namely green communication, gain more and more prominence due to energy shortage and greenhouse effect [12]. This letter focuses on the maximization of energy efficiency, defined as the delivered information bits per Joule harvested energy, by jointly optimizing transfer duration and transmit power. The contributions of this letter lie in three folds. First, it solves the challenging problem of long-distance wireless information and power transfer. Second, it improves the energy efficiency enormously. Third, it provides high QoS guarantee.

${ }^{*}$ Correspondence to: Henry Boger, Consultant, Senior Member of the International Society of Automation, Birmingham, Hoover, USA, E-mail: hwboger@msn.com

Received: July 18, 2018; Accepted: July 23, 2018; Published: July 27, 2018 
The rest of this letter is organized as follows. We first give an overview of wireless information and power transfer in large-scale MIMO systems in Section II, and then derive an energy-efficient resource allocation scheme by maximizing the efficiency [13-27].

\section{Conclusion}

A major contribution of this letter is the introduction of the largescale MIMO technique into wireless information and power transfer. By exploiting the advantage of the large-scale MIMO, this letter realizes long-distance and QoS guaranteed wireless information and power transfer. Considering the demand for green communications, an energy-efficient resource allocation scheme is proposed by jointly optimizing transmit power and transfer duration. Numerical results confirm the effectiveness of the proposed scheme.

\section{References}

1. Zhang F, Hackworth SA, Liu X, Chen H, Sclabassi RJ, et al. (2009) Wireless energy transfer platform for medical sensors and implantable devices. Conf Proc IEEE Eng Med Biol Soc 2009: 1045-1048. [Crossref]

2. Huang K, Larsson EG, Simultaneous information and power transfer for broadband wireless systems.

3. Watfa MK, Al-Hassanieh H, Selman S (2011) "Multi-hop wireless energy transfer in WSNs," IEEE Commun. Lett., 15: 1275-1277.

4. Varshney LR, (2008) "Transporting information and energy simultaneously," in Proc. IEEE Int. Symp. Inf. Theory (ISIT), 1612-1616.

5. Gozalvez J (2007) “Witricity-the wireless power transfer,” IEEE Veh. Tech. Magazine, 2: $38-44$.

6. Grover P, Sahai A (2010) Shannon meets Tesla: wireless information and power transfer," in Proc. IEEE Int. Symp. Inf. Theory (ISIT), 2363- 2367.

7. Zhang R, Ho CK (2011) "MIMO broadcasting for simultaneous wireless information and power transfer," in Proc. IEEE Globecom, pp. 1-5.

8. Xiang Z, Tao M (2012) "Robust beamforming for wireless information and power transmission," IEEE Wireless Commun. Lett., 1: 372-375.

9. Chen X, Yuen C, Zhang Z (2013) "Wireless energy and information transfer tradeoff for limited feedback multi-antenna systems with energy beamforming," IEEE Trans. Veh. Tech.

10. Marzetta TL (2010) "Noncooperative cellular wireless with unlimited number of base station antennas," IEEE Trans. Wireless Commun. 9: 3590-3600.
11. Rusek F, Persson D, Lau BK, Larsson EG, Marzetta,O TL (2013) "Scaling to MIMO opportunities and challenges with very large arrays," IEEE Signal Process. Mag., 20: 40-60.

12. Chu F, Chen K, Fettweis G (2012) "Green resource allocation to minimize receiving energy in OFDMA cellular systems," IEEE Commun. Lett., 16: 372-374.

13. Hochwald BM, Marzetta TL, Tarokh V (2004) "Multiple-antenna channel hardening and its implications for rate-feedback and scheduling," IEEE Tran. Inf. Theory, 50: 1893-1909.

14. X. Chen, Z. Zhang, S. Chen, and C. Wang, (2012) "Adaptive mode selection for multiuser MIMO downlink employing rateless codes with QoS provisioning," IEEE Trans. Wireless Commun., 11: 790- 799.

15. D. W. K. Ng, E. S. Lo, and R. Schober (2012) "Energy-efficient resource allocation for secure OFDMA systems," IEEE Trans. Veh. Tech., 61: 6: 2572-2585.

16. Dinkelbach W (1967) "On nonlinear fractional programming," Manage. Sci., 13: $492 \mathrm{C} 498$.

17. Grover P, Sahai A (2010) "Shannon meets Tesla: wireless information and power transfer," in Proc. IEEE Int. Symp. Inf. Theory (ISIT), pp. 2363- 2367.

18. Zhang R, Ho CK (2011) "MIMO broadcasting for simultaneous wireless information and power transfer," in Proc. IEEE Globecom, pp. 1-5.

19. Xiang Z,Tao M (2012) "Robust beamforming for wireless information and power transmission,” IEEE Wireless Commun. Lett., 1: 372-375.

20. Chen X, Yuen C, Zhang Z, "Wireless energy and information transfer tradeoff for limited feedback multi-antenna systems with energy beamforming," IEEE Trans. Veh. Tech., 2013.

21. Marzetta TL (2010) "Noncooperative cellular wireless with unlimited number of base station antennas," IEEE Trans. Wireless Commun., 9: 3590-3600.

22. Rusek F, Persson D, Lau BK, Larsson EG, Marzetta TL, et al. (2013) "Scaling to MIMO: opportunities and challenges with very large arrays," IEEE Signal Process. Mag., 20: 40-60.

23. Chu F, Chen K, Fettweis G (2012) "Green resource allocation to minimize receiving energy in OFDMA cellular systems," IEEE Commun. Lett., 16: 372-374.

24. Hochwald BM, Marzetta TL, Tarokh V (2004) "Multiple-antenna channel hardening and its implications for rate-feedback and scheduling," IEEE Tran. Inf. Theory, 50: 1893-1909.

25. Chen X, Zhang Z, Chen S, Wang C (2012) "Adaptive mode selection for multiuser MIMO downlink employing rateless codes with QoS provisioning," IEEE Trans. Wireless Commun., 11: 790- 799.

26. D. W. K. Ng, E. S. Lo, and R. Schober (2012) "Energy-efficient resource allocation for secure OFDMA systems," IEEE Trans. Veh. Tech., 61: 2572-2585.

27. Dinkelbach W (1967) "On nonlinear fractional programming," Manage. Sci., 13 492 C498.

Copyright: (C2018 Boger HW. This is an open-access article distributed under the terms of the Creative Commons Attribution License, which permits unrestricted use, distribution, and reproduction in any medium, provided the original author and source are credited. 